\title{
Spinal cord injury after traumatic spine fracture in patients with ankylosing spinal disorders
}

\author{
Frederik R. Teunissen, BSc, Bianca M. Verbeek, MD, Thomas D. Cha, MD, MBA, and \\ Joseph H. Schwab, MD, MS
}

Department of Orthopaedic Surgery, Massachusetts General Hospital, Harvard Medical School, Boston, Massachusetts

\begin{abstract}
OBJECTIVE Spinal cord injury (SCI) is a major complication of spinal fractures in patients with ankylosing spondylitis (AS) and diffuse idiopathic skeletal hyperostosis (DISH). Due to the uncommon nature of these conditions, existing literature consists of relatively small case series without detailed neurological data. This study aims to investigate the incidence, predictors, and sequelae of $\mathrm{SCl}$ in patients with a traumatic fracture of the ankylosed spine.
\end{abstract}

METHODS The study included all patients older than 18 years of age with AS or DISH who presented to two affiliated tertiary care centers between January 1, 1990, and January 1, 2016, and had a traumatic fracture of the spine. Factors associated with $\mathrm{SCl}$ after traumatic fracture were compared using Fisher's exact tests. Logistic regression was used for the analysis of predictive factors for $\mathrm{SCl}$. For the comparison of probability of survival between patients with and without $\mathrm{SCl}$, Kaplan-Meier methodology was used.

RESULTS One hundred seventy-two patients with a traumatic fracture of an ankylosed spine were included. Fifty-seven patients (34.1\%) had an $\mathrm{SCl}$ associated with the fracture. The cervical spine was the most fractured region for patients both with $(77.2 \%)$ and without $(51.4 \%) \mathrm{SCl}$. A cervical fracture (odds ratio $[\mathrm{OR}] 2.70, p=0.024)$ and a spinal epidural hematoma (SEH) after fracture (OR 2.69, $p=0.013$ ) were predictive of SCl. Eleven patients $(19.3 \%)$ with SCl had delayed $\mathrm{SCl}$ (range 8-230 days). Of 44 patients with SCl and sufficient follow-up, 20 (45.5\%) had neurological improvement after treatment. Early and late complication rates were significantly higher $(p=0.001$ and $p=0.004)$ and hospital stay was significantly longer $(p=0.001)$ in patients with $\mathrm{SCl}$. The probability of survival was significantly lower in the $\mathrm{SCl}$ group compared with the non-SCI group $(p=0.006)$.

CONCLUSIONS The incidence of $\mathrm{SCl}$ was high after fracture of the spine in patients with $\mathrm{AS}$ and DISH. Predictive factors for $\mathrm{SCl}$ after fracture were a fracture in the cervical spine and an $\mathrm{SEH}$ following fracture. One-fifth of the patients with $\mathrm{SCl}$ had delayed $\mathrm{SCl}$. Patients with $\mathrm{SCl}$ had more complications, a longer hospital stay, and a lower probability of survival. Less than half of the patients with $\mathrm{SCl}$ showed neurological improvement.

https://thejns.org/doilabs/10.3171/2017.5.SPINE1722

KEY WORDS ankylosing spondylitis; diffuse idiopathic skeletal hyperostosis; spinal cord injury; spine fracture; neurologic improvement; mortality

A NKYLOSING spondylitis (AS) and diffuse idiopathic skeletal hyperostosis (DISH) are diseases both leading to ankylosis of the spine, resulting in a rigid, fracture-prone spine. Because of their similarities the two are often grouped together in the literature..$^{5,19,23}$ However, AS and DISH are different diseases and have a different morphology of ankylosis., ${ }^{7,12,16}$
AS is a rheumatic inflammatory disease, affecting predominantly (90.3\%) human leukocyte antigen-B27 positive patients, $64 \%$ of whom are men. ${ }^{9}$ About $80 \%$ of the patients experience onset of symptoms before the age of 30 years. Chronic inflammation of the axial skeleton in these patients eventually results in ankylosis of the facet joints, ligaments, and intervertebral space. Patients with

ABBREVIATIONS AIS = ASIA Impairment Scale; AS = ankylosing spondylitis; $\mathrm{ASIA}=$ American Spinal Injury Association; $\mathrm{BMI}=$ body mass index; $\mathrm{CCI}=\mathrm{Charlson}$ Comorbidity Index; $\mathrm{Cl}=$ confidence interval; $\mathrm{DISH}=$ diffuse idiopathic skeletal hyperostosis; $I Q R$ = interquartile range; $\mathrm{OR}=$ odds ratio; $\mathrm{SCI}=$ spinal cord injury; $\mathrm{SEH}=$ spinal epidural hematoma.

SUBMITTED January 7, 2017. ACCEPTED May 12, 2017

INCLUDE WHEN CITING Published online October 6, 2017; DOI: 10.3171/2017.5.SPINE1722. 
AS generally show marginal syndesmophyte formation, leading to the classic "bamboo spine" radiographic appearance. . $^{16,24}$

DISH, in contrast to AS, is a noninflammatory disease, but the cause of DISH remains unknown. DISH generally manifests over the age of 50 and occurs roughly twice as often in men. Like AS, DISH causes bone proliferation resulting in ankylosis of the spine. However, patients with DISH generally show nonmarginal syndesmophyte formation, ossification of the anterior longitudinal ligament, and no involvement of the sacroiliac joint. ${ }^{16,24}$

Spinal cord injury (SCI) is a common and serious complication after traumatic fracture of the ankylosed spine and has a large impact on morbidity and mortality. ${ }^{5,12,19}$ The rate of SCI after traumatic fractures in patients with AS and DISH ranges between 19\% and 97.3\% ${ }^{7,15,19,23}$ However, the number of patients in previous studies on spinal fractures of the ankylosed spine is low, especially with regard to SCI, and information about it is scarce. ${ }^{5,23}$ This study aims to investigate the incidence, predictors, and sequelae of SCI in patients with a traumatic fracture of the ankylosed spine.

\section{Methods \\ Study Design and Patients}

Approval of our study design was obtained from the Partners IRB. We included all patients 18 years of age and older diagnosed with AS or DISH with a traumatic fracture of the spine who presented to two affiliated tertiary care centers (Massachusetts General Hospital and Brigham and Women's Hospital) between January 1, 1990, and January 1, 2016. We identified patients by searching our institution's data registry for the ICD-9 and ICD-10 codes for both AS and DISH. All patient records were manually reviewed for diagnosis of AS or DISH and a traumatic spinal fracture. Those patients who had a pathological fracture, who had a fracture before the age of 18 , or who did not have signs and/or symptoms of AS or DISH at the time of fracture were excluded.

\section{Variables}

For each patient, data were manually collected from their electronic records regarding age, body mass index (BMI), smoking status, medical comorbidities, race, sex, region of fracture, mechanism of trauma, type of treatment, and complications. The medical comorbidities were collected for the date of the fracture and included hypertension and diabetes mellitus. The Charlson Comorbidity Index (CCI) score at the time of fracture was programmed using diagnostic codes. ${ }^{6,18}$ Region of fracture was divided into cervical, thoracic, lumbar, sacral, and multiregion groups (e.g., C7-T1 fracture included in cervical, thoracic, and multiregion group; C6-7 fracture only in cervical group). Mechanism of trauma was divided into lowimpact (e.g., fall from a sitting or standing position), and high-impact trauma (e.g., motor vehicle accident or a fall from more than 8 feet).

\section{Outcome Measures}

We calculated time to presentation, diagnosis, treat- ment, discharge, and death. We used our institution's electronic records combined with the National Death Index to obtain the date of death to calculate mortality. We manually graded the degree of SCI using the American Spinal Injury Association (ASIA) Impairment Scale (AIS) preand postinjury, at first SCI symptoms (baseline SCI), at discharge, at change of SCI grade, and at last follow-up. Patients with an AIS grade decline of 1 grade or more (such as E to D) at any point after fracture were considered SCI patients. We categorized all SCI patients as either complete (AIS Grade A) or incomplete (AIS Grade B-D) SCI. Patients with SCI who showed an improvement of at least 1 AIS grade (such as D to E) during follow-up were considered neurologically improved. Early complications included all complications that occurred within 90 days after fracture. Late complications included all complications that occurred later than 90 days after fracture. We collected spinal epidural hematoma (SEH) data following fracture as a separate variable from the complications. Delayed diagnosis was defined as fracture diagnosis after the day of trauma. We defined delayed SCI as the development of SCI more than 24 hours after trauma. We subcategorized delayed neurological decline into 2 groups. In Group 1 , patient's delay was defined as patient's presentation later than 24 hours after trauma, and in Group 2, doctor's delay was defined as diagnosis later than 24 hours after patient's presentation to the hospital. All patients using systemic steroids for more than 90 days at the moment of fracture were categorized as chronic steroid users. We defined initial fracture treatment as the definitive treatment within the first admission after fracture diagnosis.

\section{Statistical Analysis}

We used Stata software (version 13.0, StataCorp) for the statistical analysis. For bivariate analysis, Fisher's exact tests were performed for noncontinuous data. For continuous nonparametric data, Mann-Whitney U-tests were performed. Nonparametric continuous data were described with the median and the interquartile range (IQR). Predictive factors for SCI after fracture that showed a trend toward significance $(\mathrm{p}<0.100)$ were analyzed for independence in a multivariable analysis using a logistic regression model. A Kaplan-Meier survival estimate was used to compare the probability of survival between groups. A $\mathrm{p}$ value less than 0.050 was considered significant.

\section{Results}

Our search resulted in 2089 patients with a diagnosis of AS or DISH. We identified 189 traumatic fracture incidents in 172 patients. All first-time traumatic spine fractures $(n=172)$ were used for the analysis. One hundred thirty-four patients (77.9\%) were diagnosed with AS and 26 patients (15.1\%) with DISH (Table 1). For 12 patients (7.0\%) no differentiation between AS and DISH could be made, because patients had radiographic signs of both diseases (AS/DISH group). When comparing the AS and DISH groups we found no significant difference in patients' baseline characteristics (Table 1).

SCI occurred in 57 (34.1\%) of 167 patients, including $21(38.2 \%)$ with complete and $34(61.8 \%)$ with incomplete 
TABLE 1. Baseline characteristics and comparison of AS versus DISH

\begin{tabular}{|c|c|c|c|c|}
\hline Characteristic & Total $^{*}$ & AS & DISH & p Value \\
\hline No. of patients & $172(100)$ & $134(77.9)$ & $26(15.1)$ & \\
\hline Median age in yrs (IQR)† & $69.3(53.4-81.0)$ & $68.6(52.2-79.3)$ & $71.3(56.3-81.5)$ & 0.377 \\
\hline Median CCl (IQR)‡ & $1(0-3)$ & $1(0-3)$ & $0(0-3)$ & 0.243 \\
\hline Median hospital stay in days (IQR)§ & $7(3-15)$ & $7(2-15)$ & $6(3-13)$ & 0.731 \\
\hline Sex & & & & 0.374 \\
\hline Male & $146(84.9)$ & $113(84.3)$ & $24(92.3)$ & \\
\hline Female & $26(15.1)$ & $21(15.7)$ & $2(7.7)$ & \\
\hline Race & & & & 0.329 \\
\hline Caucasian & $157(91.3)$ & $123(91.8)$ & $23(88.5)$ & \\
\hline African American & $4(2.3)$ & $4(3.0)$ & $0(0.0)$ & \\
\hline Hispanic/Latino & $4(2.3)$ & $2(1.5)$ & $1(3.9)$ & \\
\hline Asian & $2(1.2)$ & $2(1.5)$ & $0(0.0)$ & \\
\hline $\mathrm{BMI}\left(\mathrm{kg} / \mathrm{m}^{2}\right)$ & & & & 0.503 \\
\hline$<25$ & $30(32.3)$ & $23(34.9)$ & $4(23.5)$ & \\
\hline $25-30$ & $24(25.8)$ & $14(21.2)$ & $6(35.3)$ & \\
\hline$>30$ & $39(41.9)$ & $29(43.9)$ & $7(41.2)$ & \\
\hline Smoking & & & & 0.674 \\
\hline Yes & $28(19.6)$ & $22(20.6)$ & $5(20.0)$ & \\
\hline Former & $69(48.3)$ & $50(46.7)$ & $14(56.0)$ & \\
\hline No & $46(32.2)$ & $35(32.7)$ & $6(24.0)$ & \\
\hline Hypertension & 120/154 (77.9) & $87 / 118$ (73.7) & $22 / 25(88.0)$ & 0.195 \\
\hline Diabetes mellitus & $50 / 156(32.1)$ & $40 / 120(33.3)$ & $6 / 25(24.0)$ & 0.480 \\
\hline Chronic steroid use & 14/127 (11.0) & 14/93 (15.1) & $0 / 24(0.0)$ & 0.071 \\
\hline \multicolumn{5}{|l|}{ Traumatic impact } \\
\hline High & $43(25.9)$ & $35(27.3)$ & $7(26.9)$ & 0.999 \\
\hline Low & $123(74.1)$ & $93(72.7)$ & $19(73.1)$ & \\
\hline \multicolumn{5}{|l|}{ Region of fracture $\prod^{* *}$} \\
\hline Cervical spine & $104(60.8)$ & $80(60.2)$ & $16(61.5)$ & 0.999 \\
\hline Thoracic spine & $72(42.1)$ & $54(40.6)$ & $12(46.2)$ & 0.666 \\
\hline Lumbar spine & $26(15.2)$ & $22(16.5)$ & $3(11.5)$ & 0.769 \\
\hline Sacral spine & $3(1.8)$ & $3(2.3)$ & $0(0.0)$ & 0.999 \\
\hline Multiregion & $32(18.7)$ & $24(18.1)$ & $5(19.2)$ & 0.999 \\
\hline Vertebrae involved in fracture & & & & 0.822 \\
\hline 1 & $61(36.8)$ & $47(36.4)$ & $10(40.0)$ & \\
\hline$\geq 2$ & $105(63.3)$ & $82(63.6)$ & $15(60.0)$ & \\
\hline \multicolumn{5}{|c|}{$\begin{array}{l}\text { All data given as number of patients }(\%) \text { unless otherwise indicated. } \\
\text { * There were } 12 \text { patients }(7.0 \%) \text { in the AS/DISH group (no differentiation between AS and DISH could be made). } \\
\text { † Age: } n=165(95.9 \%), A S=127(94.8 \%), D I S H=26(100 \%) \text {. } \\
\text { ‡ CCl: } n=132(76.7 \%), A S=97(72.4 \%), D I S H=24(92.3 \%) \text {. } \\
\S \text { Hospital stay: } n=126(73.3 \%), A S=95(70.9 \%), D I S H=22(84.6 \%) \text {. } \\
\text { I Region of fracture: } n=171(99.4 \%), A S=133(99.3 \%), D I S H=26(100 \%) \text {. } \\
\text { ** Cumulative percentage is }>100 \% \text { because some patients had a fracture involving multiple regions after a single trauma (for example, C7-T1 } \\
\text { fracture included in cervical, thoracic, and multiregion groups). }\end{array}$} \\
\hline
\end{tabular}

SCI; the baseline SCI AIS grade for 2 patients was unknown. Of the patients with SCI, $36(63.2 \%)$ had SCI within 24 hours after fracture and 11 (19.3\%) had delayed SCI. Treatment is also a concern for SCI because the fracture can displace during positioning of the patient: 6 patients $(10.5 \%)$ with SCI had SCI as a direct result of surgical intervention for their fracture (Fig. 1) ${ }^{20}$ The relative time of SCI versus the time of fracture was unknown in 4 pa- tients. There was no significant difference in the SCI rate between the AS $(n=43,33.3 \%)$ and DISH $(n=11,42.3 \%)$ groups $(\mathrm{p}=0.378)$.

\section{Fracture Characteristics in SCI Patients}

The cervical spine was the most frequently fractured region in both the non-SCI (51.4\%) and SCI patients (77.2\%), occurring mostly in C5-7 (Fig. 2). However, the cervi- 

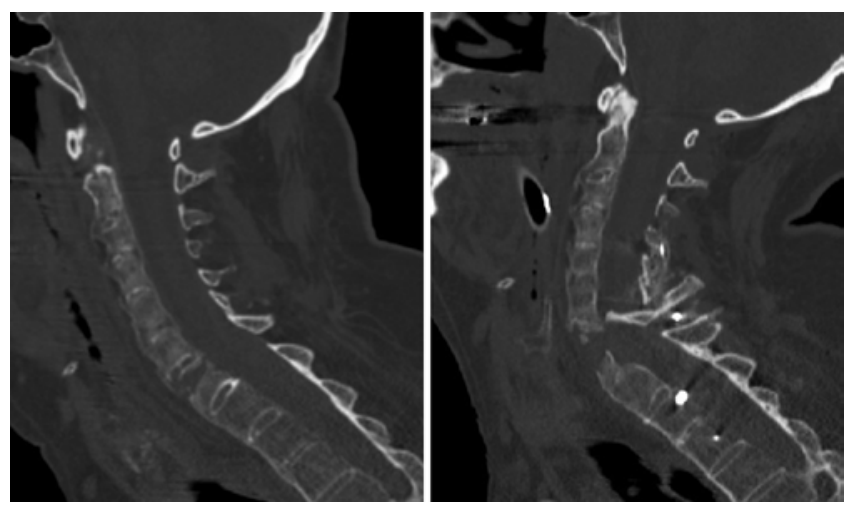

FIG. 1. Case 1. A 76-year-old man with AS presented to the hospital after a low-impact trauma, without neurological deficit (AIS Grade E). CT showed a fracture involving C6-7 (left). The patient underwent operative treatment. Immediately after surgery he suffered a complete paraplegia (AIS Grade A). CT showed canal compromise due to hyperextension of the cervical spine (right). This case highlights the importance of taking into account a rigid kyphotic cervical deformity during positioning, common in patients with AS and DISH.

cal vertebrae were more frequently fractured in patients with SCI than in patients without SCI $(\mathrm{p}=0.001$; Table 2). SEH following fracture occurred in 16 patients with an SCI (32.7\%; AS = 25 [21.6\%], DISH = 5 [22.7\%]). Logistic regression demonstrated that a fracture in the cervical region (odds ratio [OR] 2.70, 95\% confidence interval [CI] 1.24-5.87; $\mathrm{p}=0.024$ ) and an SEH following spinal fracture (OR 2.69, 95\% CI 1.14-6.34; $\mathrm{p}=0.013$ ) were independently significantly predictive for SCI.

\section{Delayed SCI}

Of all patients with delayed SCI $(\mathrm{n}=11), 4$ patients had a fracture after trauma, but were sent home without a fracture diagnosis after the initial hospital presentation; subsequently they developed SCI 8, 18, 37, and 230 days after fracture (Fig. 3). Another 3 patients did not visit a physician after a fall and developed a delayed SCI 11, 27, and 193 days after fracture. The remaining 4 patients developed SCI some time after initial fracture treatment: 3 of 4 patients after conservative treatment, 14 and 71 days after fracture (time for 1 patient unknown), and 1 patient suffered a minor fall 25 days after initial surgical fracture treatment, resulting in an SCI due to a dislocation of the anterior plate and an anterior dislocation of C-6 on C-7. The median time between fracture and delayed SCI for all patients was 26 days (IQR 14-71 days).

\section{Improvement of Neurological Function}

A total of 44 patients with SCI (77.2\%) survived the initial hospital stay, were not lost to follow-up, and had sufficient follow-up reports regarding SCI grade. The median follow-up duration after treatment for SCI of both groups was 248 days (IQR 69-1384 days). Thirty-eight patients received surgical treatment after SCI, of whom 18 patients (47.4\%) had neurological improvement after treatment. Median time to surgery after SCI was 2 days (IQR 1-13 days). Six patients were treated nonoperatively, of whom $2(33.3 \%)$ showed improvement of neurological function.

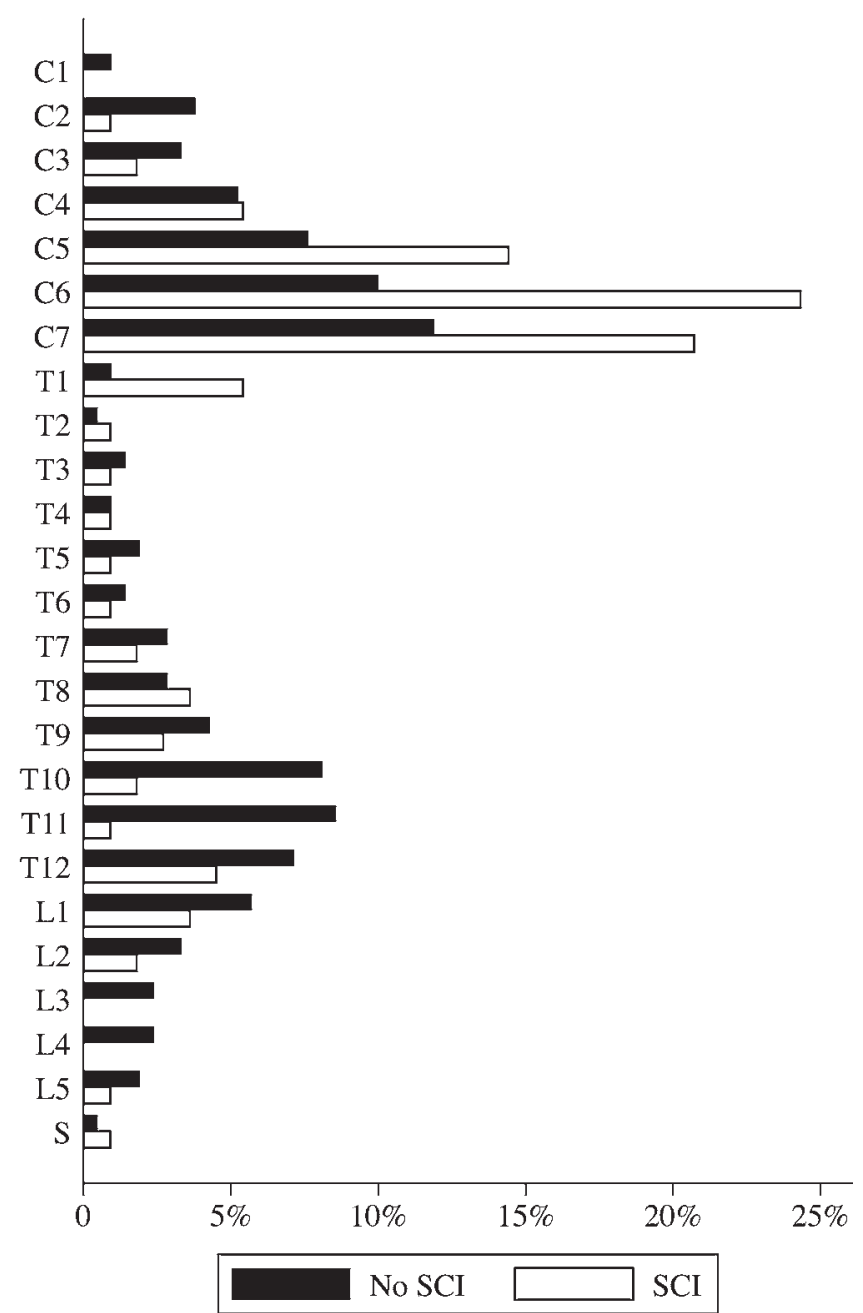

FIG. 2. Distribution of the fracture region in patients with traumatic ankylosing spine fracture, without and with SCl. Data were available for 106 patients (96.4\%) in the group without SCI, and $55(98.2 \%)$ in the group with SCl.

Of the 20 total patients who experienced neurological improvement during follow-up, 21.4\% (3 of 14) had a complete SCI at baseline and 56.7\% (17 of 30) had incomplete SCI ( $p=0.050$; Table 3$)$. The median time to neurological improvement after treatment for SCI was 35 days (IQR 12-58 days). Only 1 patient showed improvement after 3 months. This patient received only conservative treatment and had an initial AIS Grade A that improved to AIS Grade B after 7.3 years. Patients who showed improved neurological function were significantly younger (median 61.5 years) compared with patients who did not improve (median 72.8 years) during follow-up $(\mathrm{p}=0.006)$.

\section{Complications and Mortality}

Early complications occurred in 87 patients $(50.6 \%)$. The most frequent early complications were pneumonia (n $=22,12.8 \%)$, respiratory failure $(n=11,6.4 \%)$ and altered mental status $(\mathrm{n}=11,6.4 \%)$. Late complications were noted in $20(14.3 \%)$ of 140 patients with late complication data. Most frequent late complications were kyphotic deformity $(n=7,5.0 \%)$, urinary tract infection $(n=6,4.3 \%)$, 
TABLE 2. Predictive factors for $\mathrm{SCl}$ after traumatic fracture

\begin{tabular}{|c|c|c|c|}
\hline Factor & No SCl & $\mathrm{SCl}$ & $p$ Value \\
\hline No. of patients & $110(65.9)$ & 57 (34.1) & \\
\hline Median age in yrs (IQR)* & $69.0(52.1-81.4)$ & $70.9(56.1-77.6)$ & 0.836 \\
\hline Median CCI (IQR)† & $1(0-3)$ & $1(0-3)$ & 0.610 \\
\hline Diagnosis & & & 0.558 \\
\hline AS & $86(78.2)$ & $43(75.4)$ & \\
\hline DISH & $15(13.6)$ & $11(19.3)$ & \\
\hline AS/DISH & $9(8.2)$ & $3(5.3)$ & \\
\hline Sex & & & 0.823 \\
\hline Male & $94(85.5)$ & $48(84.2)$ & \\
\hline Female & $16(14.6)$ & $9(15.8)$ & \\
\hline $\mathrm{BMI}\left(\mathrm{kg} / \mathrm{m}^{2}\right)$ & & & 0.916 \\
\hline$<25$ & $20(31.3)$ & $9(33.3)$ & \\
\hline $25-30$ & $17(26.6)$ & $6(22.2)$ & \\
\hline$>30$ & $27(42.2)$ & $12(44.4)$ & \\
\hline Smoking & & & 0.351 \\
\hline Yes & $18(18.6)$ & $10(22.7)$ & \\
\hline Former & $45(46.4)$ & $24(54.6)$ & \\
\hline No & $34(35.1)$ & $10(22.7)$ & \\
\hline Hypertension & $78 / 96(81.3)$ & $38 / 53(71.7)$ & 0.217 \\
\hline Diabetes mellitus & 29/97 (29.9) & $21 / 54(38.9)$ & 0.283 \\
\hline Chronic steroid use & $9 / 82(11.0)$ & $4 / 41(9.8)$ & 0.999 \\
\hline Traumatic impact & & & 0.258 \\
\hline High & $31(29.3)$ & $11(19.6)$ & \\
\hline Low & $75(70.8)$ & $45(80.4)$ & \\
\hline \multicolumn{4}{|l|}{ Region of fracture $\ddagger \S$} \\
\hline Cervical spine & $56(51.4)$ & 44 (77.2) & 0.001 \\
\hline Thoracic spine & $51(46.8)$ & $20(35.1)$ & 0.186 \\
\hline Lumbar spine & $19(17.4)$ & $7(12.3)$ & 0.501 \\
\hline Sacral spine & $1(0.9)$ & $2(3.5)$ & 0.271 \\
\hline Multiregion & $19(17.4)$ & $13(22.8)$ & 0.414 \\
\hline $\begin{array}{l}\text { Vertebrae involved in } \\
\text { fracture }\end{array}$ & & & 0.164 \\
\hline 1 & $42(39.6)$ & $15(27.3)$ & \\
\hline$\geq 2$ & $64(60.4)$ & $40(72.7)$ & \\
\hline SEH following fracture & 13/97 (13.4) & $16 / 49$ (32.7) & 0.008 \\
\hline
\end{tabular}

All data given as number of patients (\%) unless otherwise indicated.

* Age: $\mathrm{no} \mathrm{SCl}=104(94.5 \%), \mathrm{SCl}=56(98.4 \%)$.

$\dagger \mathrm{CCl}$ : $\mathrm{no} \mathrm{SCl}=88(80.0 \%), \mathrm{SCl}=39(68.4 \%)$.

$\ddagger$ Region of fracture: no SCI = $109(99.7 \%), \mathrm{SCl}=57(100 \%)$.

$\S$ Cumulative percentage is $>100 \%$ as some patients had a fracture involving multiple regions after a single trauma (for example, a C7-T1 fracture included

in the cervical, thoracic, and multiregion groups).

and wound infection $(\mathrm{n}=3,2.1 \%)$. Bivariate analysis revealed that patients with SCI were more likely to be initially treated surgically than conservatively $(n=43,75.4 \%$; $\mathrm{p}=0.001)$ and had a significantly higher rate of early (n $=44,77.2 \% ; \mathrm{p}=0.001)$ and late $(\mathrm{n}=12 / 43,27.9 \% ; \mathrm{p}=$ $0.004)$ complications compared with patients without SCI. Patients with SCI also had a longer hospital stay (median 14 days, IQR 7-19 days) compared with those without SCI (median 6 days, IQR 1-11 days; $\mathrm{p}=0.001$ ).


FIG. 3. Case 2. A 56-year-old man with DISH presented to the hospital after a motor vehicle accident, without neurological deficit (AIS Grade E). Only radiography was performed-despite persistent back pain-on which a spinal fracture was missed (left). After 230 days, CT was performed for pulmonary embolism screening, on which an incidental fracture involving T-8 was diagnosed (right). By that time, the patient had developed a mild neurological deficit (AIS Grade D). This case promotes evaluation by CT of AS and DISH patients with persistent back pain after trauma.

Eighteen patients (10.5\%) died during the initial hospital stay after fracture, 26 patients $(15.1 \%)$ died within 3 months after fracture, and 41 patients $(23.8 \%)$ died within 1 year after fracture. The mortality rate at the initial hospital stay was significantly higher $(\mathrm{p}=0.043)$ in patients with $\mathrm{SCI}(\mathrm{n}=9,15.8 \%)$ compared with those without SCI $(\mathrm{n}=$ $6,5.5 \%)$, showed a trend toward significance $(\mathrm{p}=0.060)$ at 3 months ( $\mathrm{SCI}=12$ [21.1\%] vs non-SCI $=11[10.0 \%])$ and was significantly higher $(\mathrm{p}=0.001)$ at 1 year $(\mathrm{SCI}=$ 22 [38.6\%] vs non-SCI = 16 [14.6\%]). For 3 patients who died during the initial hospital stay, the AIS grade was not assessable. A Kaplan-Meier survival estimate analysis showed a significantly lower probability of survival in patients with SCI compared with patients without SCI ( $p=0.006$; Fig. 4). Additionally, a Kaplan-Meier survival estimate analysis showed a significantly lower probability of survival in patients with complete SCI compared with those with incomplete SCI $(\mathrm{p}=0.001)$.

We found no significant differences between the AS and DISH groups in early ( $48.5 \%$ and $50.0 \%$, respectively; $\mathrm{p}=0.999)$ and late complication rates $(11.9 \%$ and $11.5 \%$, respectively; $p=0.999)$. There was also no difference in mortality rate during the initial hospital stay $(\mathrm{AS}=14$ [10.5\%] vs DISH $=1$ [3.9\%], $\mathrm{p}=0.469$ ), at 3 months (AS $=20$ [14.9\%] vs DISH $=2$ [7.7\%], $\mathrm{p}=0.534$ ), and at 1 year $(\mathrm{AS}=33[24.6 \%]$ vs DISH $=2[7.7 \%], \mathrm{p}=0.069)$.

\section{Discussion}

Although different entities, both AS and DISH lead to an ankylosed spine, susceptible to fracture. ${ }^{5,7,22} \mathrm{We}$ also found patients with AS and DISH to be very homogenous in terms of demographics and fracture characteristics. One-third (34.1\%) of the patients in our cohort had SCI after traumatic fracture. Patients with a cervical fracture and 
TABLE 3. AIS grade improvement from first symptoms to last follow-up

\begin{tabular}{cccccc}
\hline AIS Grade & \multicolumn{5}{c}{ AIS Grade at Last Follow-Up } \\
\cline { 2 - 6 } at 1st SCl & A & B & C & D & E \\
\hline A & 11 & 2 & 1 & & \\
\hline B & & & & 1 & \\
\hline C & & & 3 & 2 & \\
D & & & & 10 & 14 \\
\hline
\end{tabular}

Bold values represent patients with no change from first $\mathrm{SCl}$ to $\mathrm{SCl}$ at last follow-up.

a fracture complicated by an SEH had a significantly higher chance of SCI. SCI was associated with an increased complication and mortality rate. Patients with SCI had a lower probability of survival compared with those without SCI. Twenty patients with SCI demonstrated neurological improvement, which occurred in the first 3 months after injury for all but 1 case.

Our finding of an SCI rate of $34.1 \%$ is lower than the average of $77.5 \%$ reported by the largest meta-analysis on traumatic spinal fractures in patients with ankylosing spinal disorders. ${ }^{23}$ Lukasiewicz et al. describe a large cohort of 939 patients with AS and a spinal fracture and reported an SCI rate of $21.2 \%$. However, their data were extracted from predefined variables in the National Inpatient Sample database, so SCI and other data may be underreported or inaccurate. ${ }^{15}$ The two largest single-institution cohorts on the subject by Caron et al. $(\mathrm{n}=112)$ and Schiefer et al. $(\mathrm{n}=$ 81) combined AS and DISH patients and reported an SCI incidence of $58 \%$ and $19 \%$, respectively. ${ }^{5,19}$ Several previous studies were reported by specialized trauma centers, so the difference noted in SCI incidence may reflect differences in referral patterns between the centers.

A delay in fracture diagnosis is often observed in this patient population $(\mathrm{n}=65,44.2 \%)$. Patients with ankylosing spinal disorders are known to have chronic back pain; therefore they might ignore the increase of pain following seemingly minor trauma. Because of the bone changes in the spine, radiological fracture assessment can be difficult, especially in the thoracic spine, and fractures easily missed. When harboring a missed and unstable fracture, these patients can develop a delayed SCI. Doctor's delay was associated with SCI in 4 cases $(2.3 \%)$ and patient's delay was associated with SCI in 3 cases (1.8\%). Persistent pain after a fall should promote further evaluation using CT. ${ }^{2,11}$

SEH following fracture was found to be predictive for the development of SCI and was noted in $21.6 \%$ of the patients with AS and in $22.7 \%$ of the patients with DISH. This is much higher than the occurrence of SEH in spinal fractures in the general population $(0.5 \%-7.5 \%) .{ }^{17}$ Contrary to a fracture causing immediate SCI, SEH can cause a subacute SCI developing within hours. The exact origin of SEH after spinal fracture remains unclear, but bleeding from the spinal epidural venous plexus is believed to be the source of the majority of the hematomas. GarzaMercado et al. suggest that firm adherence of the dural sac to the posterior longitudinal ligament may be a protective factor for rupture of the ventral venous plexus. ${ }^{10} \mathrm{How}-$

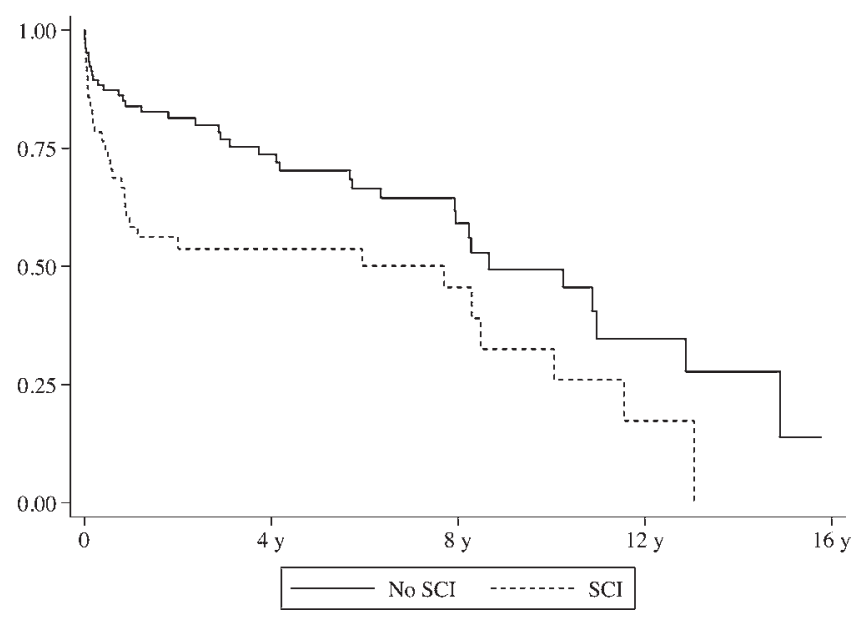

FIG. 4. Kaplan-Meier curve demonstrating survival estimates for patients with traumatic ankylosing spine fracture without $\mathrm{SCl}$ compared with patients with $\mathrm{SCl}(\mathrm{p}=0.006)$. $y=$ years.

ever, ossification of the posterior longitudinal ligament is frequently noted in both AS and DISH patients and may make the adherence to the dural sac and the venous plexus more susceptible to rupture when the spine-and possibly the posterior longitudinal ligament-is fractured..$^{16}$

Thirty patients (17.4\%) had an SEH after fracture. In 12 cases the SEH was detected on CT. Of those cases, 7 SEHs were confirmed on consecutive MRI, 4 SEHs were confirmed during immediate operative treatment following CT, and in 1 case no additional imaging or surgery was performed after initial CT. For 16 patients no SEH was observed on initial CT, but only on consecutive MRI. In 1 case the SEH was not noted on CT or MRI, but only during surgery, and in 1 case the SEH diagnosis was made at an outside hospital using unknown imaging. In addition to the literature, our findings promote MRI as the preferred tool for the detection of SEHs, because an SEH is easily missed on CT. Possible confounders of SEHs are spinal subdural hematomas and nontraumatic spinal lesions such as tumors. However, MRI can differentiate between SEH, spinal subdural hematoma, and nontraumatic spinal lesions. ${ }^{1,4,13}$

Early recognition and decompression of SEHs within 12 hours is reported to result in better neurological outcomes in the general population. ${ }^{14}$ Three case reports emphasize these findings in the ankylosing spine fracture population by describing full recovery of paraplegic and tetraplegic patients after early ( $<12$ hours) decompressive surgery. ${ }^{10,26,27}$ Fehlings et al. and Wilson et al. address the effect of early decompression on the improvement of neurological function after spinal trauma without SEH in the general population and have proved early decompression ( $<24$ hours) to be beneficial in this group. ${ }^{8,25}$ Fehlings et al. report a neurological improvement rate in traumatic cervical fracture patients after early and late surgical treatment of $43.2 \%$ and $37.0 \%$, respectively, for the complete SCI group at baseline and $63.2 \%$ and $54.7 \%$, respectively, for the incomplete SCI group at baseline. ${ }^{8}$ The majority of the 38 patients who received surgery after SCI in our cohort received surgery later than 24 hours after SCI. Only $18.2 \%$ of the patients who had a complete SCI at baseline showed 
improvement after surgery compared with $59.3 \%$ of the incomplete SCI at baseline group. The question remains as to what extent early surgical treatment influences neurological improvement for both fractures with and without $\mathrm{SEH}$ in the ankylosed spine population, especially for patients with a worse AIS grade at baseline.

We found that patients with improved neurological function were significantly younger and had an incomplete SCI significantly more often compared with the patients who did not improve. However, early death could be an important confounding factor. We found a significant lower probability of survival for patients with complete SCI compared with incomplete SCI. In addition, older age is also known to be a predictive factor for mortality; ${ }^{5,19}$ this means that if death occurs within the median time to improvement (35 days), our results could be biased.

Westerveld et al. suggest that surgical treatment may be favorable for patients with an ankylosing spinal fracture, as it may be associated with a lower mortality rate and may lead to neurological improvement more frequently. ${ }^{23}$ This is consistent with the findings of Caron et al., who also report a significant lower 1-year mortality rate in surgically treated patients $(\mathrm{p}=0.005)$ compared with nonsurgically treated patients. However, we found a significantly higher 1 -year mortality rate $(p=0.049)$ in surgically treated patients and no significant difference in neurological improvement between surgical and conservative treatment ( $p$ $=0.673$ ). Because of the small sample size and the multiple possible confounding factors, it is hard to draw conclusions from these findings. Another important difference is that Caron et al. did not find significantly higher (1-year) mortality in patients with SCI compared with patients without SCI $(p=0.91)$, in contrast to our findings $(p=0.001) .^{5}$

This study also has some limitations. First, due to the retrospective study design, we encountered some missing data and complications, and medical comorbidities are sometimes underreported in the medical reports. But because both the missing data and the underestimation of complications happened throughout the entire database, the ratio between groups should remain unchanged. As a result, we expect no different outcome in comparative analysis. Second, for the 3-month and 1-year mortality analyses, we also included the patients without 3-month and 1-year follow-up evaluations. Additionally, there is a delay in the report of death records in the National Death Index. This may have led to a minor deviation in the number and percentages of 3-month and 1-year mortality rates. The Kaplan-Meier survival estimates are already corrected for these factors. Third, for most patients the AIS grade was not mentioned in the reports and had to be manually deducted from the neurological reports by the authors. The AIS can be a broad grading scale; it is possible to have clinical neurological improvement but to remain in the same AIS grade. This can be the cause of an underestimation of the improvement of neurological function in patients. In contrast, it is possible to show improvement on the AIS without a change in ambulatory status. Twelve $(60.0 \%)$ of 20 patients who demonstrated neurological improvement were wheelchair bound after fracture and SCI, and before improvement. Seven of these patients $(58.3 \%)$ converted to ambulatory with a cane or walker and 4
(33.3\%) remained wheelchair bound; for 1 patient $(8.3 \%)$ the ambulatory status was unknown after neurological improvement. Lastly, several papers describe the difficulty in diagnosing AS and DISH. ${ }^{3,16,21}$ It is possible that patients with AS are diagnosed with DISH and vice versa, but the fracture characteristics and treatment remain similar.

\section{Conclusions}

SCI was a major complication of traumatic spinal fracture in patients with AS and DISH, occurring in one-third of the cases. A cervical fracture and an SEH were found to be predictive factors for SCI. One-fifth of the patients with SCI had delayed SCI. SCI after fracture resulted in a higher mortality and complication rate. Less than half of the patients with SCI had neurological improvement after treatment. A significantly lower probability of survival was noted in patients with an SCI. With our findings we hope to give physicians better insights into the incidence, predictors, and sequelae of SCI in patients with a traumatic fracture of the ankylosing spine.

\section{References}

1. Al-Mutair A, Bednar DA: Spinal epidural hematoma. J Am Acad Orthop Surg 18:494-502, 2010

2. Anwar F, Al-Khayer A, Joseph G, Fraser MH, Jigajinni MV, Allan DB: Delayed presentation and diagnosis of cervical spine injuries in long-standing ankylosing spondylitis. Eur Spine J 20:403-407, 2011

3. Braun J, Sieper J: Ankylosing spondylitis. Lancet 369:13791390,2007

4. Braun P, Kazmi K, Nogués-Meléndez P, Mas-Estellés F, Aparici-Robles F: MRI findings in spinal subdural and epidural hematomas. Eur J Radiol 64:119-125, 2007

5. Caron T, Bransford R, Nguyen Q, Agel J, Chapman J, Bellabarba C: Spine fractures in patients with ankylosing spinal disorders. Spine (Phila Pa 1976) 35:E458-E464, 2010

6. Charlson ME, Pompei P, Ales KL, MacKenzie CR: A new method of classifying prognostic comorbidity in longitudinal studies: development and validation. J Chronic Dis 40:373383, 1987

7. Einsiedel T, Schmelz A, Arand M, Wilke HJ, Gebhard F, Hartwig E, et al: Injuries of the cervical spine in patients with ankylosing spondylitis: experience at two trauma centers. J Neurosurg Spine 5:33-45, 2006

8. Fehlings MG, Vaccaro A, Wilson JR, Singh A, W Cadotte D, Harrop JS, et al: Early versus delayed decompression for traumatic cervical spinal cord injury: results of the Surgical Timing in Acute Spinal Cord Injury Study (STASCIS). PLoS One 7:e32037, 2012

9. Feldtkeller E, Khan MA, van der Heijde D, van der Linden S, Braun J: Age at disease onset and diagnosis delay in HLAB27 negative vs. positive patients with ankylosing spondylitis. Rheumatol Int 23:61-66, 2003

10. Garza-Mercado R: Traumatic extradural hematoma of the cervical spine. Neurosurgery 24:410-414, 1989

11. Harrop JS, Sharan A, Anderson G, Hillibrand AS, Albert TJ, Flanders A, et al: Failure of standard imaging to detect a cervical fracture in a patient with ankylosing spondylitis. Spine (Phila Pa 1976) 30:E417-E419, 2005

12. Jacobs WB, Fehlings MG: Ankylosing spondylitis and spinal cord injury: origin, incidence, management, and avoidance. Neurosurg Focus 24(1):E12, 2008

13. Jamjoom ZAB: Acute spontaneous spinal epidural hematoma: the influence of magnetic resonance imaging on diagnosis and treatment. Surg Neurol 46:345-349, 1996 
14. Lawton MT, Porter RW, Heiserman JE, Jacobowitz R, Sonntag VK, Dickman CA: Surgical management of spinal epidural hematoma: relationship between surgical timing and neurological outcome. J Neurosurg 83:1-7, 1995

15. Lukasiewicz AM, Bohl DD, Varthi AG, Basques BA, Webb ML, Samuel AM, et al: Spinal fracture in patients with ankylosing spondylitis: cohort definition, distribution of injuries, and hospital outcomes. Spine (Phila Pa 1976) 41:191-196, 2016

16. Olivieri I, D'Angelo S, Palazzi C, Padula A, Mader R, Khan MA: Diffuse idiopathic skeletal hyperostosis: differentiation from ankylosing spondylitis. Curr Rheumatol Rep 11:321328, 2009

17. Pan G, Kulkarni M, MacDougall DJ, Miner ME: Traumatic epidural hematoma of the cervical spine: diagnosis with magnetic resonance imaging. Case report. J Neurosurg 68:798-801, 1988

18. Quan H, Sundararajan V, Halfon P, Fong A, Burnand B, Luthi JC, et al: Coding algorithms for defining comorbidities in ICD-9-CM and ICD-10 administrative data. Med Care 43:1130-1139, 2005

19. Schiefer TK, Milligan BD, Bracken CD, Jacob JT, Krauss WE, Pichelmann MA, et al: In-hospital neurologic deterioration following fractures of the ankylosed spine: a singleinstitution experience. World Neurosurg 83:775-783, 2015

20. Sciubba DM, Nelson C, Hsieh P, Gokaslan ZL, Ondra S, Bydon A: Perioperative challenges in the surgical management of ankylosing spondylitis. Neurosurg Focus 24(1):E10, 2008

21. Taurog JD, Chhabra A, Colbert RA: Ankylosing spondylitis and axial spondyloarthritis. N Engl J Med 374:2563-2574, 2016

22. Werner BC, Samartzis D, Shen FH: Spinal fractures in patients with ankylosing spondylitis: etiology, diagnosis, and management. J Am Acad Orthop Surg 24:241-249, 2016

23. Westerveld LA, Verlaan JJ, Oner FC: Spinal fractures in patients with ankylosing spinal disorders: a systematic review of the literature on treatment, neurological status and complications. Eur Spine J 18:145-156, 2009

24. Whang PG, Goldberg G, Lawrence JP, Hong J, Harrop JS, Anderson DG, et al: The management of spinal injuries in patients with ankylosing spondylitis or diffuse idiopathic skeletal hyperostosis: a comparison of treatment methods and clinical outcomes. J Spinal Disord Tech 22:77-85, 2009

25. Wilson JR, Singh A, Craven C, Verrier MC, Drew B, Ahn $\mathrm{H}$, et al: Early versus late surgery for traumatic spinal cord injury: the results of a prospective Canadian cohort study. Spinal Cord 50:840-843, 2012

26. Wong $\mathrm{AS}, \mathrm{Yu} \mathrm{DH}$ : Cervical spine fracture in a patient with ankylosing spondylitis causing a C2-T9 spinal epidural hematoma-treatment resulted in a rapid and complete recovery from tetraplegia: case report and literature review. Asian J Neurosurg 10:53, 2015

27. Wu CT, Lee ST: Spinal epidural hematoma and ankylosing spondylitis: case report and review of the literature. J Trauma 44:558-561, 1998

\section{Disclosures}

Dr. Cha reports receiving grants from the North American Spine Society and the Gordon and Betty Moore Foundation; and being a consultant for GE Healthcare, Bio2, NuVasive, and K2M. Dr. Schwab reports being a speaker for Stryker Spine.

\section{Author Contributions}

Conception and design: all authors. Acquisition of data: Teunissen, Verbeek. Analysis and interpretation of data: Teunissen, Verbeek. Drafting the article: Teunissen. Critically revising the article: Verbeek, Cha, Schwab. Reviewed submitted version of manuscript: all authors. Approved the final version of the manuscript on behalf of all authors: Teunissen. Statistical analysis: Teunissen, Verbeek. Administrative/technical/material support: Cha, Schwab. Study supervision: Schwab.

\section{Correspondence}

Frederik R. Teunissen, Department of Orthopaedic Surgery, Spine Service, Massachusetts General Hospital, Harvard Medical School, Yawkey Bldg., 55 Fruit St., Ste. 3A, Boston, MA 02114. email: freekteunissen@gmail.com. 